\title{
Sabine Anselm
}

Forschungsstelle Werteerziehung und Lehrerbildung, Ludwig-Maximilians-Universität München

\section{Christian Hoiß}

Zertifikatsprogramm „el mundo - Bildung für nachhaltige Entwicklung im Lehramt“, Ludwig-Maximilians-Universität München

\section{Digital, diskursiv, partizipativ}

\section{Die Materialien der BNE-BOX als fachdidaktische Zugänge einer Bildung für nachhaltige Entwicklung}

\section{DOI: https://doi.org/10.53349/sv.2021.i1.a38}

\begin{abstract}
Der vorliegende Beitrag beschäftigt sich mit der fachdidaktischen Einbindung von Bildung für nachhaltige Entwicklung (BNE) in Unterrichtskontexte. Im Fokus stehen dabei die partizipativ mit Lehramtsstudierenden entwickelten Unterrichtsmaterialien der digitalen Plattform „BNE-BOX“. Die BNE-BOX reagiert damit auf eine grundlegende Problematik: Einerseits wird der Ruf nach einer umfassenden Einbindung von BNE in schulische Kontexte immer lauter, andererseits stellen sowohl die Reflexion des Konzepts BNE als auch die fachdidaktische Einbindung der Inhalte und Methoden für Lehrpersonen eine enorme Herausforderung dar. Denn die Übersetzung sowie die Reduktion der interdisziplinären Wissensbestände in das jeweilige Fach und die darin üblichen Denkmuster und Forschungsparadigmen sind hoch komplex. Die BNE-BOX liefert Ansätze, wie diese fachdidaktische Übersetzung unabhängig von Alter und Lerngruppe diskursiv geleistet werden kann.
\end{abstract}

BNE, Fachdidaktik, digitale Materialien, SDGs, diskursiv

\section{Herausforderung BNE}

Bildung für nachhaltige Entwicklung (BNE) schafft für Menschen einen Raum, jene Werte, Kenntnisse und Fertigkeiten zu erwerben, die für eine zukunftsfähige Gestaltung des eigenen Lebens und der Gesellschaft notwendig sind (vgl. DUK 2011, 9f.). Daher wurden in den letzten dreißig Jahren seitens der Vereinten Nationen massive Anstrengungen unternommen, um die Umsetzung von BNE auf nationaler Ebene voranzutreiben. Zugleich gilt es aus pädagogischer Sicht zu bedenken, dass BNE nicht in Top-down-Prozessen vorgeschrieben werden 
sollte. Vielmehr gilt es, Bildungspozesse so anzulegen, dass sie von den Einzelpersonen ausgehen. Um den damit verbundenen Anforderungen angemessen zu begegnen, orientiert sich die BNE-BOX an drei pädagogisch-didaktischen Grundprinzipien: dem Diskursprinzip nach Habermas, dem Prinzip der Interdisziplinarität mit dem Ziel einer umfassenden Multiperspektivität sowie dem Prinzip der Selbstreflexion (vgl. Anselm, Hoiß \& Vogt 2018; Hoiß 2019, S. 4151).

In diesem Kontext ist es grundlegend wichtig, Diskursfähigkeit für die Verhandlung normativer Konzepte wie der Nachhaltigkeit einzuüben. Daraus leitet sich der dezidiert interdisziplinäre Ansatz der BNE-BOX ab. Um Problemstellungen des globalen Wandels sowie ihrer regionalen und lokalen Ausprägungen umfassend integrieren zu können (vgl. LeNa 2014, S. 2), müssen ökologische, soziale, ökonomische und kulturelle Aspekte von Nachhaltigkeit gleichermaßen berücksichtigt sowie natur- und humanwissenschaftliche Disziplinen miteinander verknüpft werden. Für die Lösung der globalen Probleme gilt es also, die Innovationskraft aller Disziplinen miteinzubinden und zu nutzen. Dies lässt sich auch auf den Schulbereich übertragen: Jedes Fach kann aus den eigenen Kompetenz- und Tätigkeitsfeldern heraus einen Beitrag zur interdisziplinären Aufgabe BNE leisten.

\section{Digitale Unterrichtsmaterialien}

Die BNE-BOX ist eine digitale Plattform für fachdidaktisch konzipierte Lehr-Lernmaterialien im BNE-Kontext (vgl. Abb. 1). Jede Kategorie ist mit konkreten Unterrichtsvorschlägen gefüllt, die je nach Bedarf einzeln oder in Kombination für den eigenen Fachunterricht verwendet und den situativen Kontexten entsprechend angepasst werden können. Zu Beginn jeder Einheit findet sich eine Kurzbeschreibung der Unterrichtsidee, daneben gibt es Angaben zur empfohlenen Altersgruppe, Schulart und Gruppengröße sowie Vorschläge zu Unterrichtsfächern, in denen eine Verwendung möglich erscheint. Es folgen umfassende Hintergrundinformationen, die Lehrpersonen im Sinne einer Sachanalyse einen fundierten Einblick in die doch oft sehr komplexen Zusammenhänge der BNE-Themen geben. Danach wird der Ablauf der Unterrichtseinheit in Einzelschritten beschrieben; dafür benötigte Materialien oder Links sind zur einfachen Handhabung direkt verlinkt. Am Ende jeder Einheit findet sich zudem eine schwerpunktmäßige Zuordnung zu relevanten Gestaltungskompetenzen. 


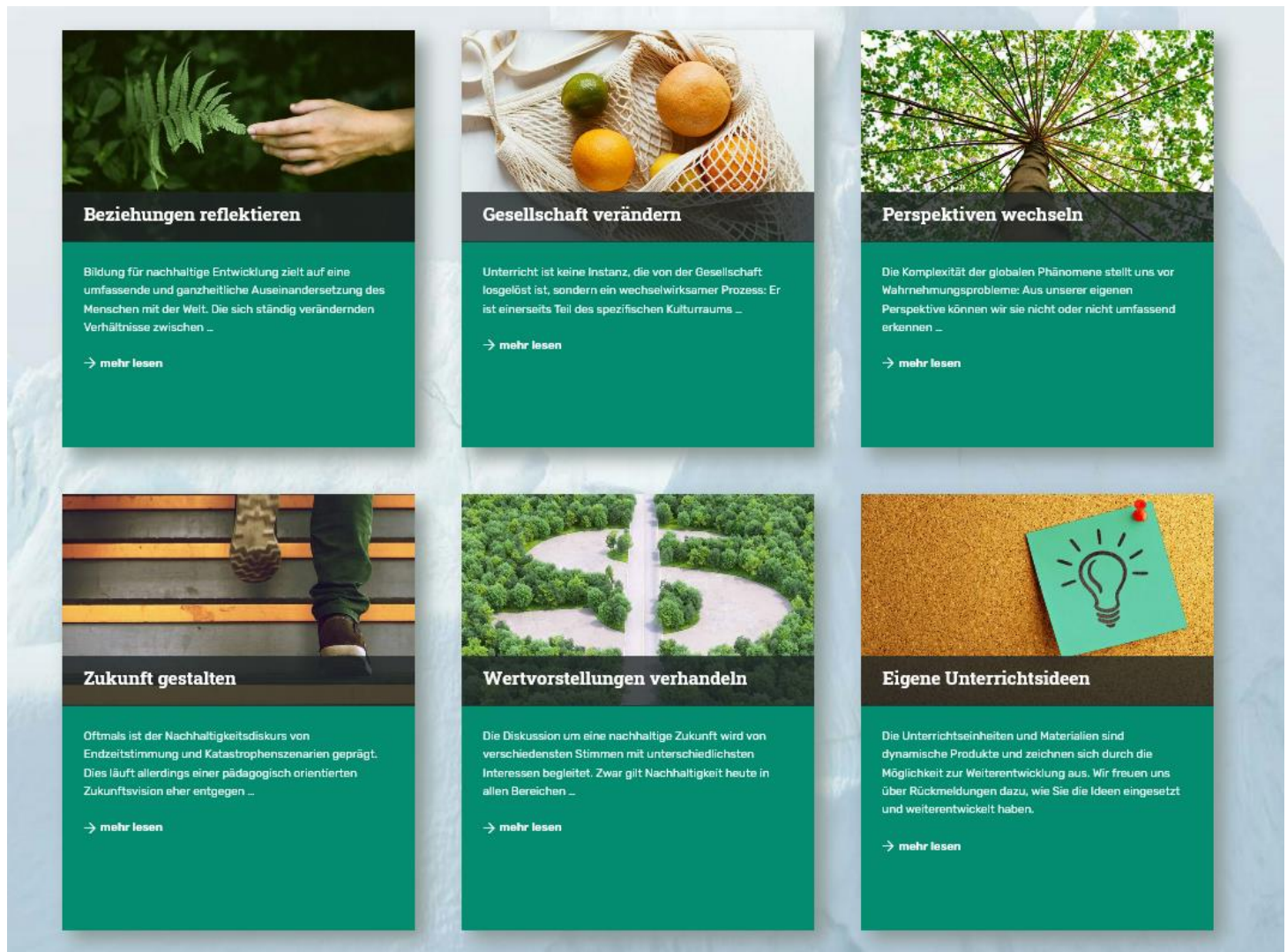

Abb. 1: Thematische Kategorien der Unterrichtsideen in der BNE-BOX | Foto: BNE-BOX

Parallel zu den Unterrichtsmaterialien finden sich in einer eigenen Kategorie Methodenmuster, die nach formalen Kriterien konzipiert wurden. Zu den Methodenmustern sind jeweils konkrete thematische Materialien zugeordnet, die zeigen, wie die Methoden im Fachunterricht eingebunden werden können.

\section{Partizipation und Kollaboration}

Der BNE-BOX liegt ein grundlegend partizipativer Ansatz zugrunde: Ein beträchtlicher Teil der Lehr-Lernmaterialien wurde gemeinsam mit Studierenden der Ludwig-Maximilians-Universität München in Lehramtsveranstaltungen des Projektes „DISKURS-ARENA: Nachhaltigkeit und Ethik in der Lehrerbildung" (vgl. Anselm, Hoiß \& Vogt 2018) entwickelt. ${ }^{1}$ In sog. Unterrichtswerkstätten integrierten die Seminarteilnehmenden BNE-Ansätze im eigenen Fach (Umfang pro Einheit ca. 90 Minuten); diese Unterrichtseinheiten wurden im Seminarkontext vorgestellt, mit den anderen Seminarteilnehmenden diskutiert und nach mehreren Feedbackrunden (auch mit den Dozierenden) im Schulkontext durchgeführt. Die integrative Arbeit mit 
professionellen Unterrichtsaufzeichnungen der Teilnehmenden (vgl. Hoiß 2019, S. 49-53) ermöglichte wichtige Impulse für die Reflexion der Gesprächs- und Kommunikationsprozesse bei den Studierenden und führte letztlich zu einer kontinuierlichen Weiterentwicklung der Lehr-Lernmaterialien bis zur Publikation in der BNE-BOX.

Dabei wird deutlich: Die BNE-BOX ist kein statisches Produkt. Sie zeichnet sich vielmehr durch kooperative Offenheit und die Möglichkeiten zur Weiterentwicklung aus (vgl. Abb. 1, 6. Box). Alle Lehrpersonen können interaktiv konkrete Ideen, Vorschläge oder eigene Unterrichtseinheiten einreichen und in einem diskursiven und feedback-orientierten Review-Prozess mit dem Redaktionsteam unter eigenem Namen veröffentlichen. Dies entspricht einem Kernanliegen der BNE-BOX, aktuelle Unterrichtsinhalte kooperativ zu gestalten und kontinuierlich weiterzuentwickeln. Getreu den Leitlinien der BNE-BOX - miteinander reden, weiter denken, gemeinsam handeln - entsteht im Diskurs mit Bildungsakteur*innen ein Netzwerk, das über die Grenzen der virtuellen BNE-BOX hinaus auch in die Strukturen von Bildungseinrichtungen hineinwirkt.

\section{Literaturverzeichnis}

Anselm, S., Hoiß, C. \& Köppel, M. (2021). BNE-BOX - Fachdidaktisch konzipierte Lehr-Lernmaterialien für diskursiv gestalteten Unterricht in allen Fächern. www.bne-box.de, Stand vom 19.05.2021.

Anselm, S., Hoiß, C. \& Vogt, M. (2018). DISKURS-ARENA. Nachhaltigkeit und Ethik in der Lehrerbildung. In Walter Leal Filho (Hg.): Nachhaltigkeit in der Lehre. Theorie und Praxis der Nachhaltigkeit (S. 37-49). Berlin: Springer Spektrum.

DUK - Deutsche UNESCO-Kommission (2011). UN-Dekade ,Bildung für nachhaltige Entwicklung' 20052014. Nationaler Aktionsplan für Deutschland. Stand: September 2011. Bonn: UNESCO.

de Haan, G., Kamp, G., Lerch, A., Müller-Christ, G., Martignon, L. \& Nutzinger, H. G. (2008). Nachhaltigkeit und Gerechtigkeit. Grundlagen und schulpraktische Konsequenzen. Berlin, Heidelberg: Springer.

Habermas, J. (1983). Moralbewußtsein und kommunikatives Handeln. Frankfurt am Main: Suhrkamp.

Habermas, J. (1991). Erläuterungen zur Diskursethik. Frankfurt am Main: Suhrkamp.

Hoiß, C. (2019). Deutschunterricht im Anthropozän. Didaktische Konzepte einer Bildung für nachhaltige Entwicklung. München: LMU München, Elektronische Hochschulschriften. https://edoc.ub.uni-muenchen.de/24608/, Stand vom 19. Mai 2021.

LeNa - Deutschsprachiges Netzwerk LehrerInnenbildung für eine nachhaltige Entwicklung (2014). Lehrerlnnenbildung für eine nachhaltige Entwicklung - Von Modellprojekten und Initiativen zu neuen Strukturen! Ein Memorandum zur Neuorientierung von Lehrerlnnenbildung in Deutschland, Österreich und der Schweiz. Lüneburg. https://www.leuphana.de/fileadmin/user_upload/Forschungseinrichtungen/infu/personen/Stoltenberg_Ute/Memorandum_LeNa_01_09_14.pdf, Stand vom 20.05.2021. 


\section{Anmerkungen}

\footnotetext{
${ }^{1}$ Das Projekt DISKURS-ARENA sowie die BNE-BOX wurden im Rahmen der gemeinsamen Qualitätsoffensive Lehrerbildung von Bund und Ländern aus Mitteln des Bundesministeriums für Bildung und Forschung gefördert.
}

\section{Autor*innen}

\section{Sabine Anselm, Prof. ${ }^{\text {in }}$ Mag. Dr.}

Professorin für Didaktik der deutschen Sprache und Literatur. Sie leitet die Forschungsstelle Werteerziehung und Lehrerbildung an der LMU München. Ihre Forschungsschwerpunkte konzentrieren sich auf Vermittlungsprozesse von Fragen der Bildung für nachhaltige Entwicklung, auf die Reflexion ethischer und ästhetischer Fraugestellungen im Literaturunterricht sowie auf Kommunikation in Lehr-Lernkontexten.

Kontakt: sabine.anselm@Imu.de

\section{Christian Hoiß, Mag. Dr.}

Abgeordneter Lehrer und Koordinator des Zertifikatsprogramms „el mundo - Bildung für nachhaltige Entwicklung im Lehramt" an der LMU München. Seine Forschungsschwerpunkte liegen auf kulturwissenschaftlichen Zugängen im Sprach- und Literaturunterricht, mediendidaktischen Implikationen des digitalen Wandels sowie auf der Bearbeitung der Grenze zwischen einer fachdidaktischen und erziehungswissenschaftlichen BNE-Forschung.

Kontakt: christian.hoiss@Imu.de 